\title{
MiR171h restricts root symbioses and shows like its target NSP2 a complex transcriptional regulation in Medicago truncatula
}

\author{
Vinzenz Hofferek', Amelie Mendrinna', Nicole Gaude ${ }^{1}$, Franziska Krajinski ${ }^{1 *}$ and Emanuel A Devers ${ }^{1,2^{*}}$
}

\begin{abstract}
Background: Legumes have the unique capability to undergo root nodule and arbuscular mycorrhizal symbiosis. Both types of root endosymbiosis are regulated by NSP2, which is a target of microRNA171h (miR171h). Although, recent data implies that miR171h specifically restricts arbuscular mycorrhizal symbiosis in the root elongation zone of Medicago truncatula roots, there is limited knowledge available about the spatio-temporal regulation of miR171h expression at different physiological and symbiotic conditions.
\end{abstract}

Results: We show that miR171h is functionally expressed from an unusual long primary transcript, previously predicted to encode two identical miR171h strands. Both miR171h and NSP2 transcripts display a complex regulation pattern, which involves the symbiotic status and the fertilization regime of the plant. Quantitative Real-time PCR revealed that miR171h and NSP2 transcript levels show a clear anti-correlation in all tested conditions except in mycorrhizal roots, where NSP2 transcript levels were induced despite of an increased miR171h expression. This was also supported by a clear correlation of transcript levels of NSP2 and MtPt4, a phosphate transporter specifically expressed in a functional AM symbiosis. MiR171 h is strongly induced in plants growing in sufficient phosphate conditions, which we demonstrate to be independent of the CRE1 signaling pathway and which is also not required for transcriptional induction of NSP2 in mycorrhizal roots. In situ hybridization and promoter activity analysis of both genes confirmed the complex regulation involving the symbiotic status, $\mathrm{P}$ and $\mathrm{N}$ nutrition, where both genes show a mainly mutual exclusive expression pattern. Overexpression of miR171h in $M$. truncatula roots led to a reduction in mycorrhizal colonization and to a reduced nodulation by Sinorhizobium meliloti.

Conclusion: The spatio-temporal expression of miR171h and NSP2 is tightly linked to the nutritional status of the plant and, together with the results from the overexpression analysis, points to an important function of miR171h to integrate the nutrient homeostasis in order to safeguard the expression domain of NSP2 during both, arbuscular mycorrhizal and root nodule symbiosis.

Keywords: Symbiosis, Plant miRNA, miR171h, NSP2, Plant nutrition

\section{Background}

Plants constantly have to cope with phosphate $\left(\mathrm{P}_{\mathrm{i}}\right)$ limiting conditions and one strategy to overcome $\mathrm{P}_{\mathrm{i}}$ limitation is the development of a mutualistic association called arbuscular mycorrhizal symbiosis (AMS), which is formed by most land plants and fungi of the phylum Glomeromycota.

\footnotetext{
* Correspondence: Krajinski@mpimp-golm.mpg.de; edevers@ethz.ch ${ }^{1}$ Max-Planck-Institute of Molecular Plant Physiology, Am Mühlenberg 1, 14476 Potsdam, (OT) Golm, Germany

2Present address: Department of Biology, Swiss Federal Institute of Technology Zurich, Zürich, Switzerland
}

AMS can enhance phosphate uptake and growth of the plant $[1,2]$ and is named for the formation of intracellular tree like structures called arbuscules. Arbuscules are mostly formed in the inner cortical cell layer of mycorrhizal roots and are always surrounded by the plant-derived periarbuscular membrane (PAM), the site of nutrient exchange $[3,4]$. The formation of AMS is initiated after a chemical dialogue between the host plant and symbiont [5]. The plant secretes strigolactones, a group of plant hormones known to stimulate of fungal spore germination and hyphal branching [6]. In return, the fungus releases a complex mixture of lipochito-oligosaccharides, called 
Myc-LCOs or Myc-factors, which stimulate the formation of AMS and induces lateral root formation in the legume plant Medicago truncatula [7].

Strigolactone synthesis and secretion is induced by $\mathrm{P}_{\mathrm{i}}$ limitation [8-11]. Biosynthesis of strigolactones requires the GRAS type transcription factors Nodulation Signaling Pathway (NSP) 1 and NSP2, and nsp1nsp2 double mutants show a reduced colonization by mycorrhizal fungi [12]. Interestingly, NSP1 and NSP2 are key components of the Nod-factor signaling pathway leading to root nodule formation [13,14]. Recent results [7] suggest that NSP2 is also involved in AMS. The authors showed that nsp2 mutant plants do not respond to Myc-LCOs and are less colonized. Furthermore, NSP2 interacts with another GRAS transcription factor Required for Arbuscular Mycorrhization (RAM) 1, and controls the expression of the glycerol-3phosphate acyltransferase RAM2. Mutations in both genes lead to strongly reduced colonization by Rhizophagus irregularis. Interestingly, colonization with the pathogenic oomycete Phytophthora palmivora is also impaired in ram 1 and ram 2 mutants (if otherwise, need to specify) $[15,16]$. The important role of NSP2 in strigolactone biosynthesis, RNS and AMS implies that NSP2 is an integral component of the common signaling pathway [17]. Therefore, it can be expected that the spatial and temporal expression of NSP2 is tightly controlled.

Micro RNAs (miRNAs) are key regulators of gene expression and act by target transcript cleavage and/or translational repression $[18,19]$. These small non-coding RNA molecules are predominantly $21 \mathrm{nt}$ in size and have an important role in regulating developmental processes, hormonal signaling, organ polarity, RNA metabolism, and abiotic and biotic stresses of the plant [20-24]. Some miRNAs, e.g. miR166 and miR169, have also been found to be involved in root nodule symbiosis [25-27]. First evidence that miRNAs are also involved in AMS came from the observation that multiple miRNAs, e.g. miR399, are differentially regulated in the shoots and roots of mycorrhizal M. truncatula, tobacco and tomato plants [28,29]. Cloning and deep sequencing of the small RNAs and the degradome of mycorrhizal $M$. truncatula roots identified many miRNAs and their target mRNAs, of which several were differentially expressed in mycorrhizal roots [30]. Interestingly, a novel member of the miRNA171 family, miR171h, was shown to target NSP2 [30,31]. Given the above-mentioned role of NSP2, miR171h has been implicated in regulating root endosymbiosis by controlling a key component of the Sym-pathway [27]. This assumption has been strengthened by showing miRNA171h expression affects the mycorrhizal colonization, is induced by MycLCOs, and is conserved among mycotrophic plants [32]. Also, it was shown that the expression of both, miR171h and NSP2, is induced upon cytokinin treatment and that this regulation is dependent on Cytokinin Response1
(CRE1) [33]. Cytokinins and CRE1 are involved in nodule organogenesis [34], but cytokinins have also been implicated to be involved in arbuscular mycorrhizal symbiosis [35]. Additionally, a recent study employing deep sequencing of Lotus japonicus nodules revealed a non-canonical miR171 isoform, related to Medicago miR171h, which targets LjNSP2 [36]. These results indicate an additional regulating role of miR171h in nodule symbiosis, however a direct involvement could not be demonstrated so far [32,36].

In this study we show that miR171h negatively regulates both types of root endo-symbioses through perception of the nutritional status of the host plant and shows a mutually exclusive expression pattern with its target NSP2 in the root cortex of $M$. truncatula plants.

\section{Results}

Expression of an 811 bp miR171h primary transcript mediates NSP2 transcript cleavage in vivo

To confirm the functionality of the predicted $811 \mathrm{bp}$ primary transcript of miR171h [31] and the potential to silence NSP2 in vivo, we applied miRNA sensor constructs. Three different constructs were applied (Figure 1A). The first construct, MIR171h-GFP, contained an 811 bp fragment of the MIR171h primary transcript [31], which was constitutively expressed by a 35S-promoter. The construct includes an independent and constitutively expressed eGFPer as a visual transformation control. The second construct, miR171h binding site (MBS)-NSP2, represented the actual miRNA sensor. It was composed of a 35S-promoter driven $\mathrm{mRFP}$ fused to five repeats of the miR171h binding site of NSP2. As a control, the MBS of NSP2 was mutated to a scrambled sequence (MBS-mut), which was unable to bind miR171h. The constructs were used in Agrobacterium tumefaciens-mediated tobacco leaf infiltration assays and the mRFP fluorescence around the infiltration site was monitored (Figure 1B and Additional file 1: Figure S1). When MBS-NSP2 was co-infiltrated with MIR171H-GFP, the mRFP fluorescence was abolished. The fluorescence was restored when MBS-mut and MIR171H-GFP were coinfiltrated, indicating that the loss of mRFP fluorescence was specifically due to miR171h-mediated sensor cleavage. The loss of fluorescence was due to drastically reduced mRFP protein levels in MIR171H-GFP co-infiltrated tobacco leaves of MBS-NSP2 compared to MBS-mut and single infiltration (Figure $1 \mathrm{C}$ and Additional file 1: Figure S1). These results confirmed that NSP2 is regulated by miR171h through specific binding of this miRNA to its previously identified binding site and is consistent with previous degradome results [30] and RACE experiments [32].

\section{MiR171 h and NSP2 transcript levels are affected by the symbiotic status of the root and by $\mathrm{P}$ and $\mathrm{N}$ levels} Previous studies suggested that miR171h is induced in the root elongation zone of mycorrhizal roots and that 


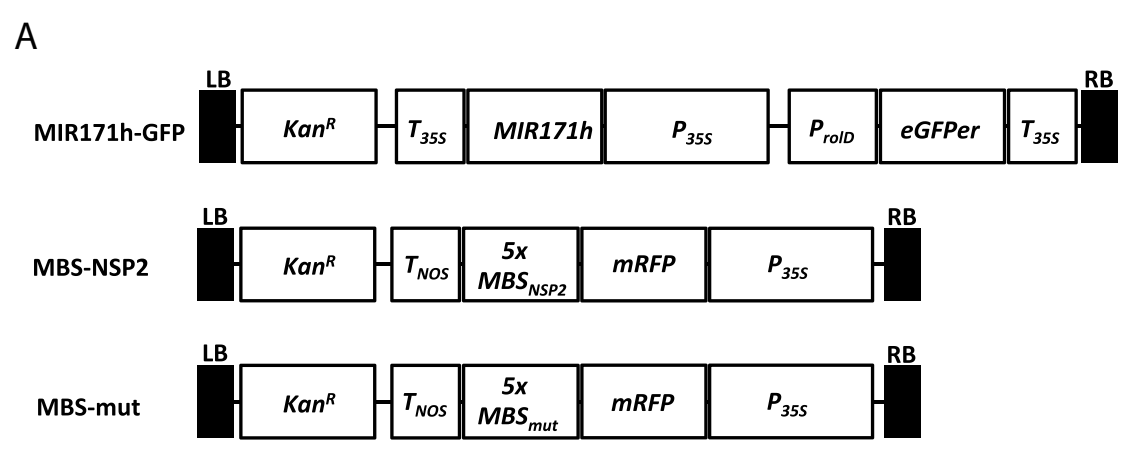

B

\begin{tabular}{lll}
\hline bright field & GFP3 fluorescence mRFP fluorescence
\end{tabular}

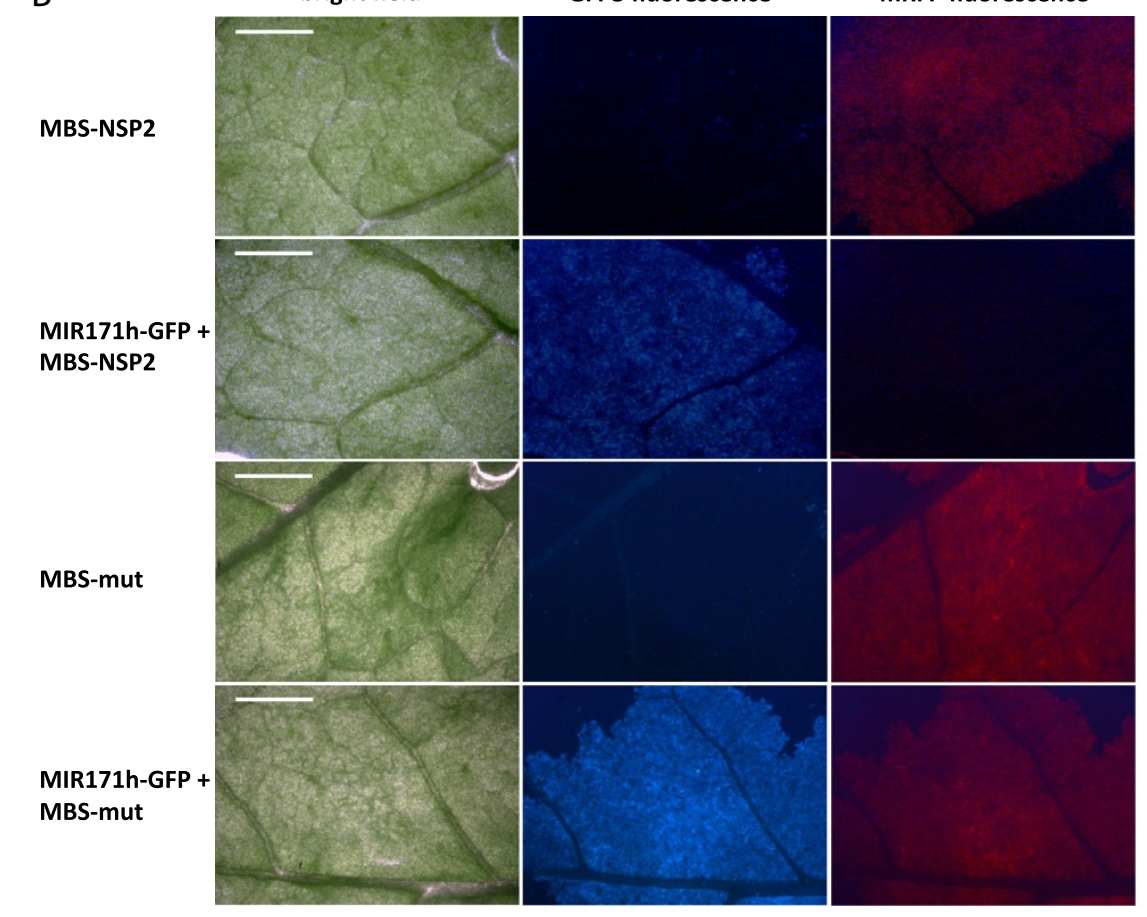

C

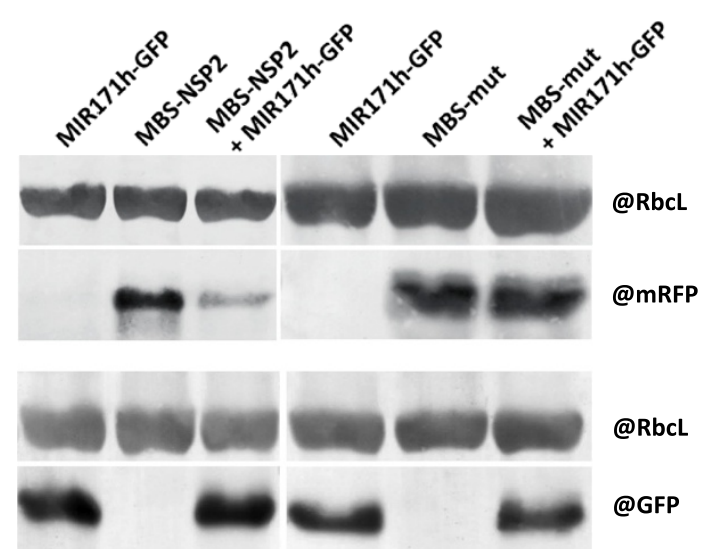

Figure 1 (See legend on next page.) 
(See figure on previous page.)

Figure 1 In vivo confirmation of NSP2 gene silencing by miR171h using MIR171h overexpression and mRFP sensor constructs.

(A) T-DNA structure of vectors used for leave infiltration experiments. MiR171h overexpression construct (MIR171h-GFP) in pK7WG2D [37] and sensor constructs with either wild-type (MBS-NSP2) or mutated miR171h (MBS-mut) binding site of NSP2 cloned in pGWB455 [38]. LB: left boarder, Kan?: kanamycin resistance gene (nptll), Tnos: nopaline synthase terminator, MIR171h: miR171h primary transcript, $\mathrm{P}_{355}$ : 355 promoter, green-fluorescent

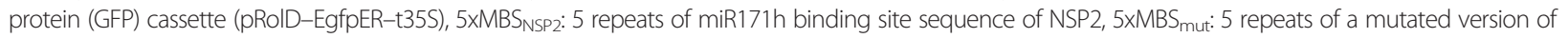
the miR171h binding site sequence of NSP2. (B) Co-infiltration of miR171 h overexpression and mRFP sensor constructs. Nicotiana benthamiana leaves were infiltrated with the two sensor constructs MBS-NSP2 or MBS-mut. For each sensor construct, co-infiltration experiments with the MIR171h-GFP construct were carried out. Note the decreased mRFP fluorescence due to miR171 h-mediated cleavage of mRFP sensor. Bright field images, GFP3 fluorescence and mRFP fluorescence are shown. Scale bar: $5 \mathrm{~mm}$. (C) Western blot to prove miR171h cleavage of the miR171h binding site within the NSP2 sequence. Proteins were extracted from leaves infiltrated with MIR171h-GFP, MBS-NSP2 or MBS-mut and co-infiltration of both constructs. The upper part of the picture shows a western blot where mRFP was detected, indicating the presence of the sensor; the lower part shows a western blot with detection of GFP, indirectly indicating the presence of miR171h. On both blots, RuBisCO proteins were detected to demonstrate equal loading of the protein samples.

NSP2 transcript levels are slightly repressed in mycorrhizal roots [32]. Also, miR171h transcription is directly induced by high phosphate nutrition [30]. To investigate the transcriptional regulation of NSP2 and miR171h in more detail, we analyzed both transcript levels in response to mycorrhizal symbiosis and root nodulation, and under different phosphate and nitrogen fertilization treatments. For this purpose we compared the abundance of mature miR171h to the relative transcript abundance of NSP2 after normalization to full nutrition $(+\mathrm{P}+\mathrm{N})$ conditions (Figure 2). As expected, miR171h accumulation was repressed by phosphate starvation $(\mathrm{P} \leq 0.05)$, i.e. positively influenced by high phosphate conditions. It also increases in nodulated roots, in which case it is further enhanced by nitrogen starvation. These results indicate that both NSP2 and MIR171h show a

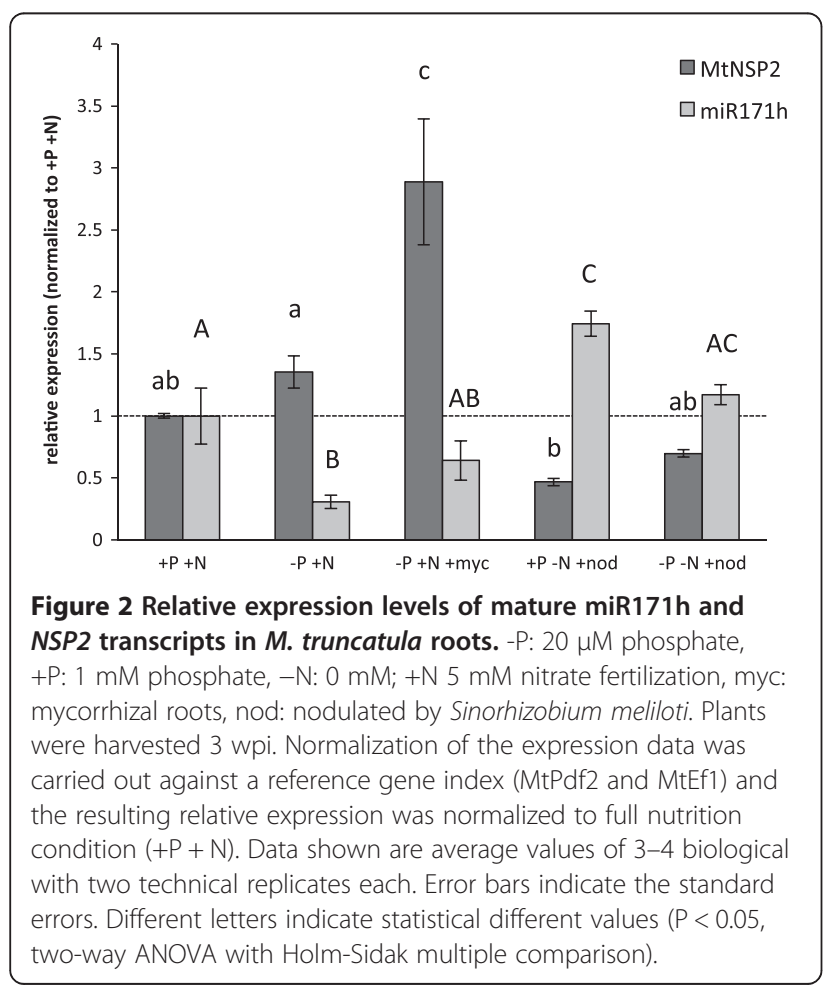

complex regulation of their transcription, which depends on the symbiotic status of the roots and the nutrient fertilization regime.

\section{NSP2 transcript levels in mycorrhizal roots are elevated despite of increased miR171 $\mathrm{h}$ expression}

A clear anti-correlation $(\mathrm{r}=-0.98 ; \mathrm{p}<0.05)$ of miR171h and NSP2 accumulation was present in all but the mycorrhizal condition (Figure 2). In the conditions used, NSP2 shows the highest relative transcript abundance in mycorrhizal roots as compared to all other conditions tested.

To investigate the miR171h and NSP2 expression in mycorrhizal roots over a time-course of AM symbiosis development, an experiment of 6 weeks was carried out and RNA accumulation of marker genes for AM symbiosis development and function were analyzed (Additional file 1: Figure S2). This clearly showed that the expression of primiR171h increases in mycorrhizal roots from 2 weeks post inoculation on, as compared to non-mycorrhizal roots under the applied conditions $(-\mathrm{P},+\mathrm{N})$. Additionally, the time-course confirms elevated NSP2 transcript levels in mycorrhizal roots, despite of enhanced miR171h accumulation. Therefore we assume that the abundance of NSP2 transcript levels in mycorrhizal roots is maintained by a miR171h-independent factor.

To further investigate the NSP2 transcript levels in mycorrhizal roots, we analyzed if NSP2 transcript level shows a correlation to MtPt4 transcript levels in individual plants. MtPt4 encodes a phosphate transporter specifically expressed in arbuscule-containing cells [39] and can be regarded as a marker for a functional AM symbiosis. We found a clear positive correlation $(\mathrm{r}=0.927, \mathrm{P}<0.01)$ of NSP2 and MtPt4 transcript levels (Figure 3 ). This supports the assumption that NSP2 is induced in mycorrhizal roots by a mycorrhiza-dependent factor.

\section{The suppression of functional symbiotic structures by high phosphate fertilization is independent of NSP2}

MiR171h transcript levels are increased in plants supplied with high phosphate concentrations. Additionally, at high phosphate conditions mycorrhizal colonization is 


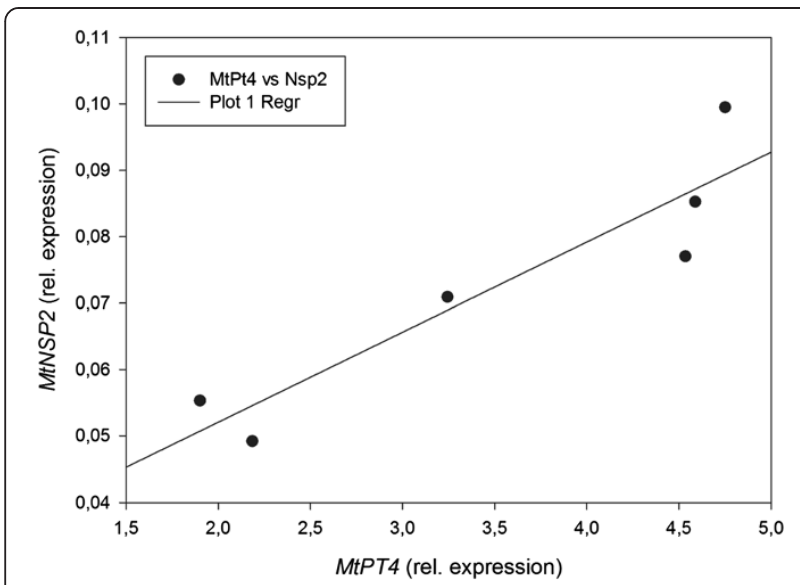

Figure 3 The relative transcript abundance of NSP2 positively correlates with MtPt4. Scatter plot of the relative expression of NSP2 against the relative expression of MtPt4 of individual WT plants including a linear regression (black line). A statistically significant correlation was calculated $(r=0.927, P<0.01$, Pearson product moment correlation). All plants were harvested 4 wpi. Normalization of the expression data was carried out against a reference gene index (MtPdf2 and MtEf1).

often decreased as compared to plants grown at phosphate starvation and additionally results in less functional symbiotic structures, namely arbuscules $[28,40]$. Therefore, is might be assumed that the suppression of mycorrhizal symbiosis at high phosphate conditions is dependent on the miR171h-NSP2 regulon. To address this question, we inoculated wild type and $n s p 2$ mutant plants with $R$. irregularis growing in phosphate starvation $\left(20 \mu \mathrm{M} \mathrm{P} \mathrm{P}_{\mathrm{i}}\right)$ or high phosphate $\left(1 \mathrm{mM} \mathrm{P} \mathrm{P}_{\mathrm{i}}\right)$ conditions. Transcript levels of MtPT4 as a marker for a functional AM symbiosis were measured at three weeks after inoculation. Mycorrhizal wild-type plants and $n s p 2$ mutants showed significantly decreased levels of MtPT4 expression when grown at high phosphate fertilization (Additional file 1: Figure S3), which indicates a significant lower frequency of functional symbiotic structures of both lines. Hence, suppression of mycorrhizal colonization by high phosphate fertilization is independent of NSP2.

\section{Induced expression of miR171h at high phosphate conditions is not dependent on CRE1}

A recent study of cis regulatory elements of the NSP2 promoter gave evidence that NSP2, as well MIR171h, transcription is directly influenced by cytokinin and depends on the cytokinin receptor CRE1 [33]. To analyze whether the induction of miR171h at high phosphate and NSP2 induction in mycorrhizal roots is dependent on CRE1-mediated cytokinin perception, we investigated the relative expression levels of miR171h and NSP2 in both conditions using cre1-1 mutant plants and wildtype control plants.
The cre1-1 mutation has subtle effect on the NSP2 transcript abundance compared to wild-type plants in regard to the increased expression in mycorrhizal plants (Figure 4B). On the one hand, only the increase of the NSP2 transcript abundance in wild-type plants is statistically significant $(\mathrm{P} \leq 0.05)$ compared to cre1-1 plants, whereas on the other hand there is no statistical significance between wild-type and cre1-1 plants at mycorrhizal conditions. This might indicate that the transcriptional induction of NSP2 in mycorrhizal roots is not directly dependent of CRE1, however we cannot rule out that NSP2 expression levels might be indirectly influenced by CRE1 during arbuscular mycorrhizal symbiosis. We also investigated the effect of cre1-1 on the AM symbiosis by monitoring the relative transcript levels of the mycorrhizal marker genes MtPt4 and RiTEF [3,41]. Expression level of both genes did not differ between mycorrhizal cre1-1 and wild type plants (Figure 4C) at 4 weeks post inoculation (wpi), which indicates that cre1-1 mutants are not impaired in mycorrhizal development. That is also supported by a similar correlation between NSP2 and MtPt4 relative transcript levels in cre1-1 plants compared to the wild type (Additional file 1: Figure S4). In contrast to the nodule symbiosis, where CRE1 is clearly involved in miR171h and NSP2 regulation [33], these results do not clearly demonstrate that the induction of NSP2 in mycorrhizal roots is directly mediated by CRE1.

Both cre1-1 and wild type plants showed a significant increase in the amount of miR171h at high $\mathrm{P}_{\mathrm{i}}$ conditions (1 $\mathrm{mM})$ compared to either non-mycorrhizal or mycorrhizal low $\mathrm{P}_{\mathrm{i}}$ conditions $(20 \mu \mathrm{M})$ (Figure $\left.4 \mathrm{~A}\right)$. At high phosphate conditions, no significant difference in the relative abundance of miR171h between cre1-1 and wild type plants could be observed, indicating that the phosphateinduced expression of miR171h is not mediated by CRE1.

\section{Reporter fusions confirm the complex and spatial regulation of NSP2- and miR171h- promoter activities}

An increased level of both NSP2 and miR171h in mycorrhizal roots suggests that these transcripts might be spatially separated in roots. We therefore used promoter-reporter fusions to localize the promoter activity of MIR171h and NSP2 in roots grown at different nutritional and symbiotic conditions. A $1248 \mathrm{bp}$ fragment upstream of the NSP2 coding sequence and a 900 bp fragment upstream of the miR171h primary transcript were fused to a $\beta$-glucuronidase (GUS) reporter gene. Both promoter-reporter constructs were transformed into M. truncatula roots by Agrobacterium rhizogenes-mediated transformation.

As expected from the transcript accumulation pattern, promoter activity pattern of both MIR171h and NSP2 showed drastic changes in response to different phosphate and nitrate fertilization treatments (Figure 5). At 

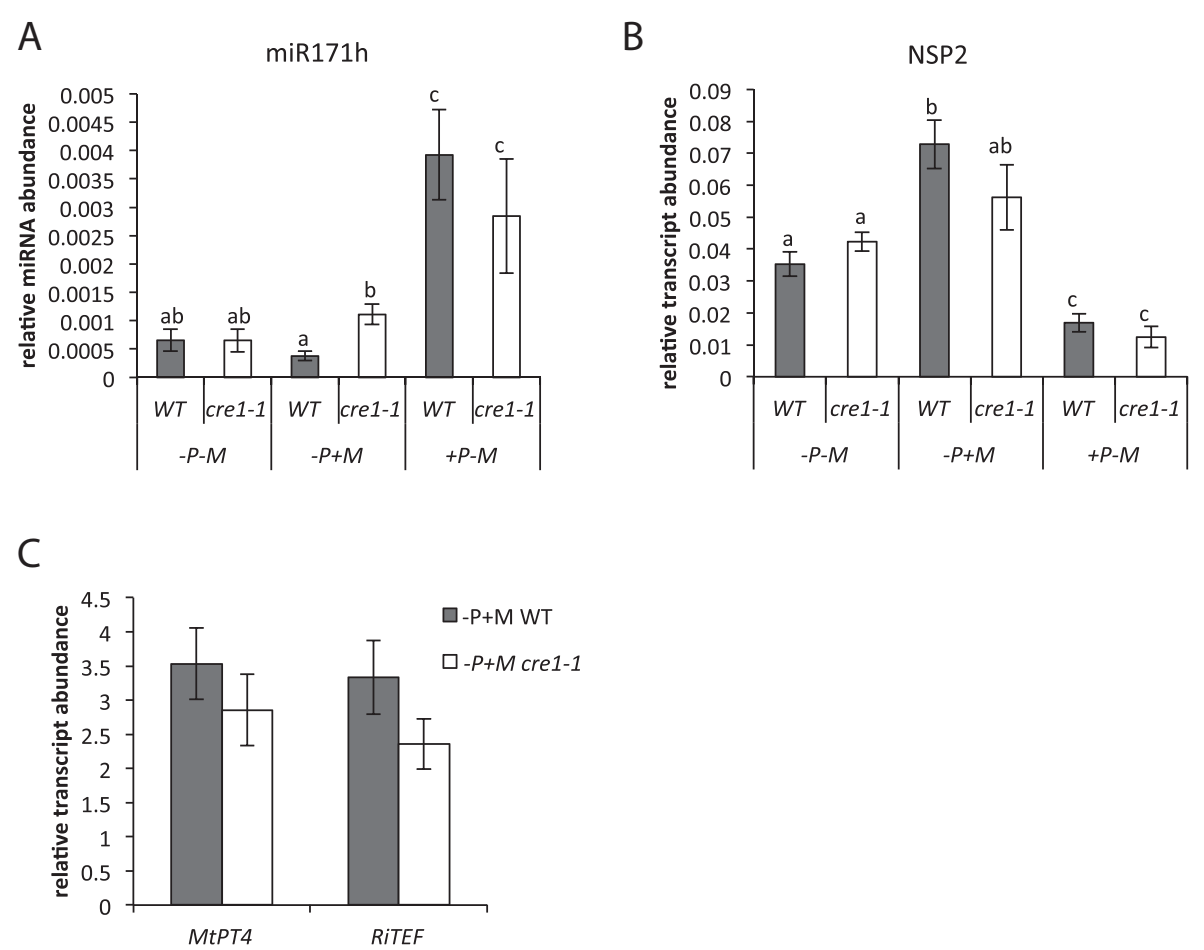

Figure 4 The cre1-1 mutation does not affect phosphate dependent regulation of miR171h as well as NSP2 transcript abundance and has no effect on mycorrhizal induced expression of NSP2 and arbuscular mycorrhizal marker genes. The relative expression of mature miR171h (A) or NSP2 (B) in roots of either WT plants (grey bars) or cre1-1 plants (white bars) determined at different phosphate fertilization and mycorrhizal conditions. -P: 20 MM phosphate, +P: 1 mM phosphate, $-\mathrm{M}$ : non-mycorrhizal roots, +M: mycorrhizal roots. (C) The relative expression of mycorrhizal the marker genes MtPt4 and RiTEF were determined in root material from the same plants shown in $\mathbf{A}$ and $\mathbf{B}(-P+M)$. All plants were harvested 4 wpi. Normalization of the expression data was carried out against a reference gene index (MtPdf2 and MtEf1). Data shown are average values of 3-6 biological replicates. Error bars indicate the standard errors. Different letters indicate statistical different values $(P<0.05)$. A and B: two-way ANOVA with Holm-Sidak multiple comparison. C: one-way ANOVA).

phosphate starvation, the promoter of MIR171h was mainly active in the central cylinder and the endodermis (Figure 5B). However, after colonization with $R$. irregularis, a weak GUS signal was detectable in distinct, but not in all, arbuscule-containing cells (Figure 5A"). The NSP2 promoter also showed a strong activity in the central cylinder and endodermis during phosphate starvation. In addition, the NSP2 promoter showed a weak activity in cortical cells of non-mycorrhizal roots (Figure 5F), whereas in mycorrhizal roots an additional strong activity was observed in the epidermal cell layer, root hairs and in some arbusculecontaining cells of the root (Figure 5E and E").

This observation might be a reasonable explanation for the above mentioned increased transcript abundance of NSP2 in mycorrhizal roots (Figure 2 and Additional file 1: Figure S2). During nitrogen starvation no major MIR171hpromoter activity could be observed except in some isolated cortical cells (Figure 5C), which might represent spontaneous promoter activity or staining artifacts. Increased miR171h expression seen during RNS (Figure 2) therefore is not due to the lack of nitrogen fertilization. In contrast to miR171h, the NSP2 promoter showed a more complex activity pattern in roots of nitrogen-starved plants. Parts of the roots showed NSP2 promoter activity in apparently random patterns in the cortex cells and endodermis (Figure 5G), whereas in different parts of the same root the promoter activity was observed in the whole cortex (Figure 5H). At full nutrition condition the MIR171h (Figure 5D) and NSP2 promoter (Figure 5I) showed contrasting localizations, where the MIR171h promoter was active in all root cells with the strongest signals in the central cylinder. In turn, the NSP2 promoter was not active in the root epidermis and cortex but active in the central cylinder and endodermis. The results of the promoter GUS study in root sections are illustrated in Additional file 1: Figure S5.

Next we analyzed the promoter activity of MIR171h and NSP2 in root nodules of plants grown at high phosphate, without nitrate and inoculated with Sinorhizobium meliloti. Representative young and mature nodules are shown in 

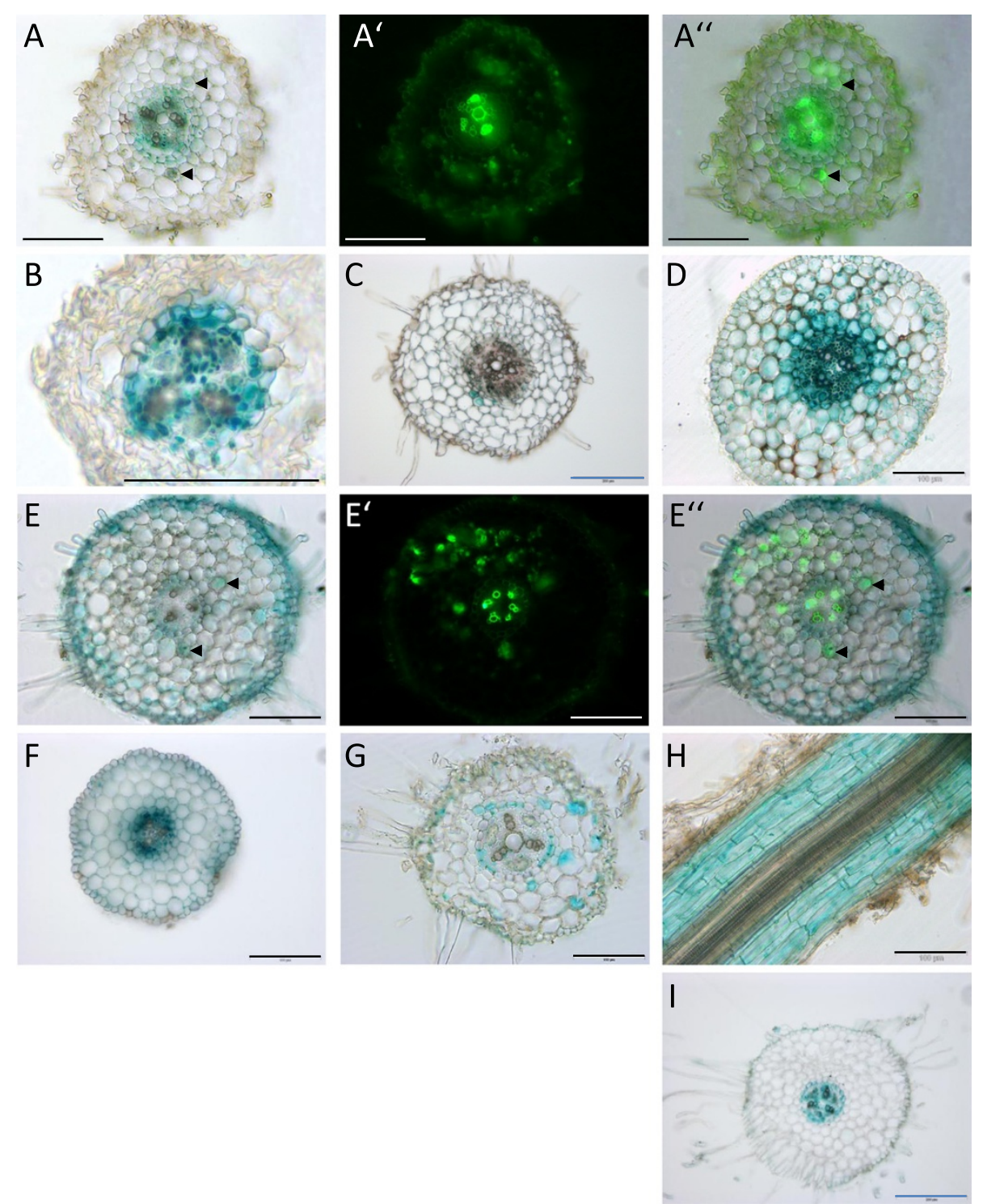

Figure 5 The NSP2 and the miR171h promoter show distinct regulation in response to nutrients and mycorrhizal colonization. Roots were transformed with promoter-uidA fusions of either the MIR171h (A-D) or the NSP2 (E-I) promoter. After root transformation, chimeric plants were potted into substrate and grown under different nutritional regimes: phosphate starvation (B and F), phosphate starvation and mycorrhizal colonization ( $\mathbf{A}$ and $\mathbf{E})$, nitrogen starvation $(\mathbf{C}, \mathbf{G}$, and $\mathbf{H})$ or full nutrition $(\mathbf{D}$ and $\mathbf{I})$. $\mathbf{A}, \mathbf{A}^{\prime}$ and $\mathbf{A}^{\prime \prime}$ as well as $\mathbf{E}, \mathbf{E}^{\prime}$ and $\mathbf{E}^{\prime}$ represent identical root sections, with $\mathbf{A}^{\prime} / \mathbf{E}^{\prime}$ showing WGA Alexafluor 488 fluorescence to visualize $R$. irregularis structures and $\mathbf{A}^{\prime \prime} / \mathbf{E}^{\prime \prime}$ showing overlay of bright filed images and WGA Alexafluor 488 fluorescence. Scale bars represent $100 \mu \mathrm{m}$ (black and white) or $200 \mu \mathrm{m}$ (blue). Arrowheads point to GUS staining in cortical cells containing arbuscules.

Figure 6. The MIR171h promoter showed only weak activity in young nodules (Figure 6A) but in mature nodules an increased activity was visible at the nodule tip with the strongest activity matching the meristematic zone and getting gradually weaker in the direction of the subsequent infection and nitrogen fixation zones (Figure 6B). It is worth mentioning that the root cortex cells flanking the nodules showed strong GUS activity, which is in contrast to non- inoculated control roots where no promoter activity was observed in the root cortex (Figure 5C).

The NSP2 promoter was active in young and mature nodules with the strongest activity in the tip of young nodules and the vascular bundles of mature nodules (Figure 6C and D). Similar to the MIR171h promoter, the NSP2 promoter was also active in the root cortex in the vicinity of young and mature nodules. 


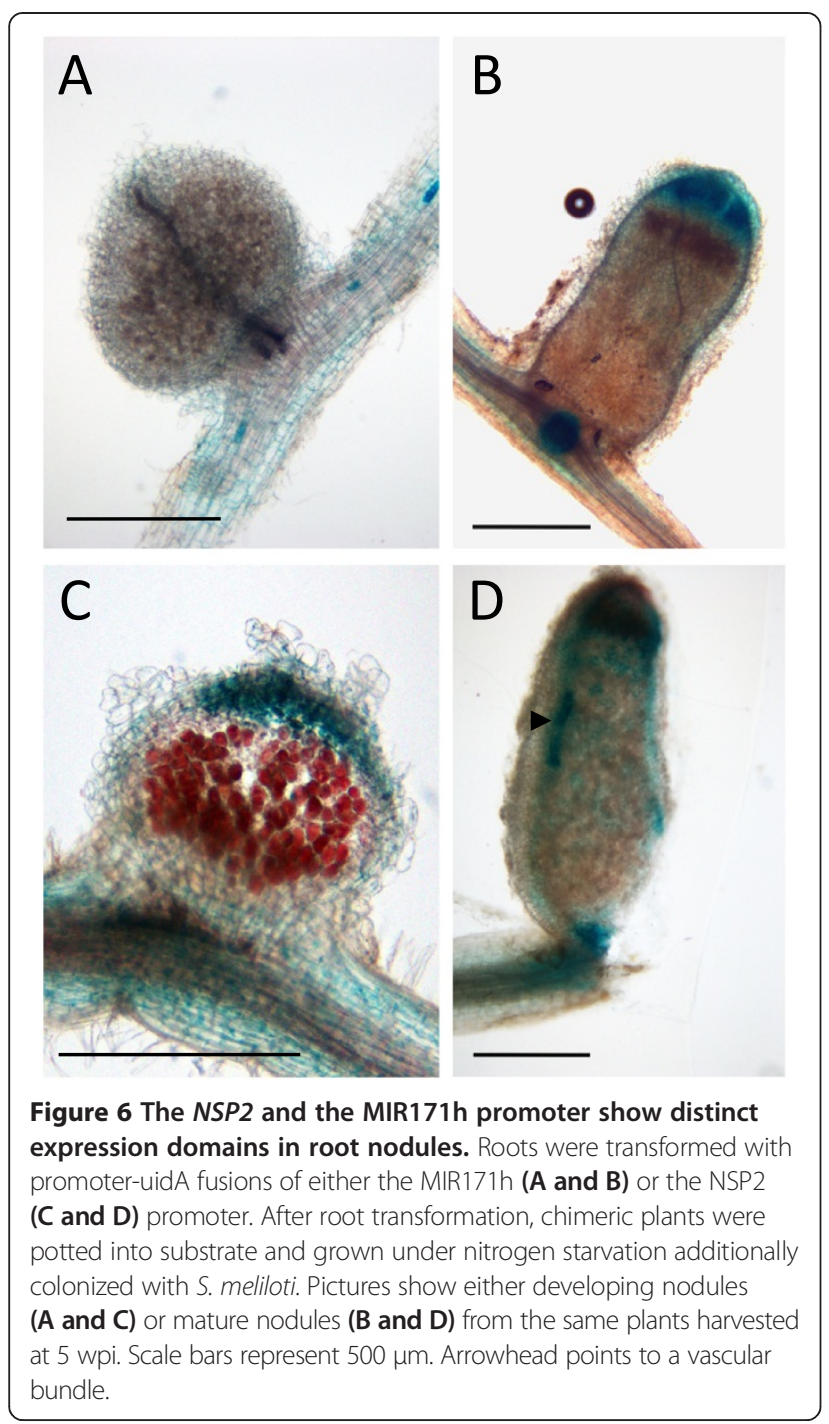

These results demonstrate that the promoter activities of NSP2 and MIR171h are mainly mutually exclusive and are affected by phosphate as well as nitrate fertilization and by root colonization with $R$. irregularis and $S$. meliloti.

\section{Mature miR171h accumulates the meristematic zone of root nodules}

To localize the site of mature miR171h accumulation in mycorrhizal and nodulated roots, we used in situ hybridization with a miR171h-specific LNA probe (Figure 7). An LNA probe with a scrambled nucleotide sequence served as a negative control. Consistent with the location of the miR171h promoter activity, we found an accumulation of mature miR171h in the central cylinder of mycorrhizal roots (Figure 7A). We found only weak signals of mature miR171h accumulation in all arbusculecontaining cells, however the MIR171h promoter activity was only observed in distinct arbuscule-containing cells (Figure 5A). Based on the low signal to background ration it is not possible to draw a clear conclusion of its localization but its likely to be in arbuscule containing cells. Localization of the mature miRNA in root nodules was in agreement with the observed promoter activity pattern in mature nodules. Mature miR171h molecules accumulated to the meristematic zone of nodules with the signal intensity gradually decreasing towards the infection zone. No miR171h accumulation could be detected in the nitrogen fixation zone (Figure $7 \mathrm{C}$ ). These results show that mature miR171h accumulation is consistent with its spatial origin of transcription and point to a protective role of miR171h against target mis-expression in root nodules and probably arbuscule containing cells.

\section{Over-expression of miR $171 \mathrm{~h}$ leads to a reduced mycorrhizal colonization and reduced nodule numbers}

To analyze the biological function of the observed spatial regulation of NSP2 and miR171h transcription, we ectopically over-expressed miR171h in $M$. truncatula roots. For this purpose, M. truncatula roots were transformed with the construct, MIR171h-GFP (Figure 1), already used for the leaf infiltration assay to drive the constitutive expression of pri-miR171h. The empty vector was used as a control. The root-transformed plants were either inoculated with $R$. irregularis or $S$. meliloti to analyze the impact of miR171h over-expression on AMS or RNS, respectively. At 5 weeks post inoculation, over-expression of primiR171h led to a very strong accumulation of mature miR171h resulting in a drastic reduction of NSP2 transcript levels (Figure 8A and B). In addition, we also observed a reduction in $M t D W A R F 27$ transcript abundance, which is a downstream target of NSP2 and important for converting the strigolactone orobanchol into didehydroorobanchol [12]. Interestingly, we found significantly reduced intraradicular rRNA level of $R$. irregularis but not of MtPt4 transcript level, which is similar to the unchanged MtPt4 transcript levels in mycorrhizal roots of nsp2-2 mutant plants (Additional file 1: Figure S4). The microscopic analysis of the mycorrhizal phenotype [42] showed a concomitant reduction in the mycorrhizal intensity to similar levels as the $n s p 2-2$ mutant line (Figure $8 \mathrm{~A}$ ), but no significant reduction of the arbuscule abundance was observed in the pri-miR171h overexpressing plants (Additional file 1: Figure S6). This implies that plant roots which over-accumulated mature miR171h were less colonized by the mycorrhizal fungus whereas arbuscule development in colonized root areas was not impaired. Additionally, we also found significantly reduced nodule numbers in roots over-expressing mature miR171h compared to the vector controls (Figure $8 \mathrm{~B}$ ).

In summary, ectopic overexpression of the primary miR171h transcript in roots of $M$. truncatula is leading to a phenotype analogous to the nsp2-2 mutant phenotype during mycorrhizal and nodule symbiosis. 

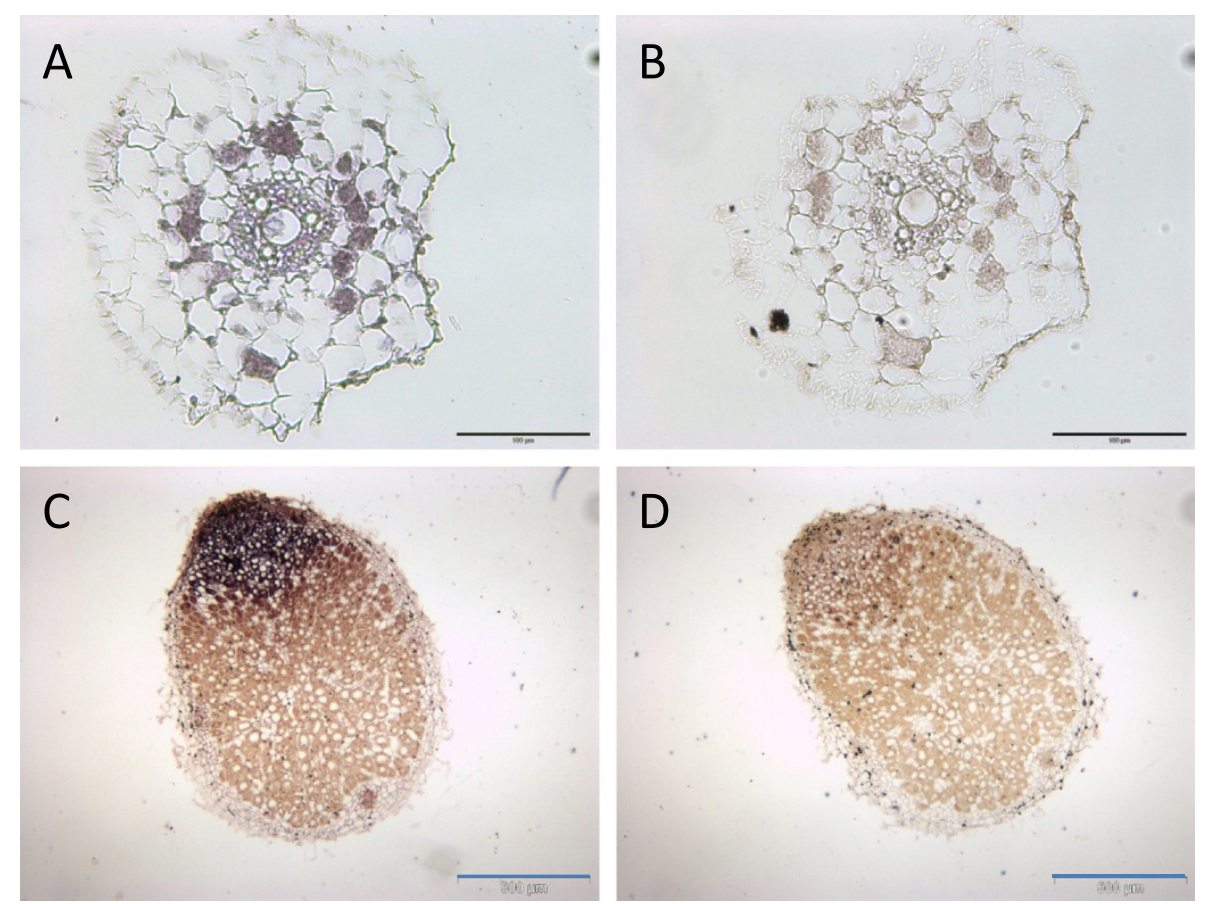

Figure 7 Localization of mature miR171h accumulation in mycorrhizal roots and root nodules via in-situ hybridization. Specific miR171h LNA probes ( $A$ and $\mathbf{C}$ ) or scramble probes ( $B$ and $\mathbf{D}$ ) were used for in situ hybridization to localize mature miR171h molecules in mycorrhizal roots (A,B) or root nodules (C,D). Detection was done using NBT/BCIP solution. Scale bars represent $100 \mu \mathrm{m}$ (black) and $500 \mu \mathrm{m}$ (blue).

\section{Discussion}

One of the recently identified miRNAs, which specifically target genes essential for root endosymbiosis is mtr-miR171h [30].

MiR171h has later been predicted to be produced from an 811 bp long primary transcript encoding two miR171h loci on a single arm of its fold-back structure [31]. In this work, we provided experimental proof that miR171h is functionally expressed from an at least 811 bp long primary transcript. Cleavage of NSP2 transcripts by miR $171 \mathrm{~h}$ has been verified by RACE-based methods [30,32,36]. However, so far functional analysis of miR171h has been carried out with the primarily identified partial precursor encoding only a single miRNA duplex [32]. By using a specific miRNA-sensor construct in a $N$. benthamiana leaf infiltration assay we could confirm in-vivo that the long double duplex-containing MIR171h primary transcript exhibits biological activity. This suggests that the long primary transcript described by Branscheid et al. [28] resembles the endogenous source of mature miR171h in M. truncatula.

The target of miR171h, NSP2, is a GRAS-type transcription factor, which is essential for the formation of root nodule symbiosis $[13,43,44]$, strigolactone biosynthesis [12] and is involved in the Myc-factor signaling pathway [7]. It has been recently shown that miR171h overexpression leads to a reduction of mycorrhizal root colonization [32] and it has been proposed to act specifically in arbuscular mycorrhizal symbiosis. Expression data of miR171h implies that this miRNA is induced by full nutrition conditions [30] and we hypothesized that miR171h is regulated by the phosphate status of the plant. Data from Arabidopsis and Rapeseed did not imply that the miR171 family is regulated by phosphate and nitrogen availability [45]. However, the data from the current study give strong evidence that the expression of miR171h is mainly induced by high phosphate availability (Figure 2). It is possible that the phosphate dependent regulation of a miR171 family member is dependent of the ability to undergo root endosymbiosis, because the miR171h isoform is only present in AMS capable plants $[27,32]$. Whether additional miR171 family members are phosphate responsive in these plant species is not known. Interestingly, the promoter GUS reporter assays suggest that the expression of miR171h is not merely regulated by phosphate per se but rather by a combination of phosphate and nitrogen levels, because the activity of the miR171h promoter is absent at high phosphate and low nitrogen conditions compared to full nutrition conditions (Figure 5C and D).

Recently, it has been shown that miR171h expression is induced by cytokinin treatment and dependent on the CRE1 pathway $[33,46]$ i.e. that the loss of CRE1 leads to loss of miR171h induction by cytokinins. Because it is 


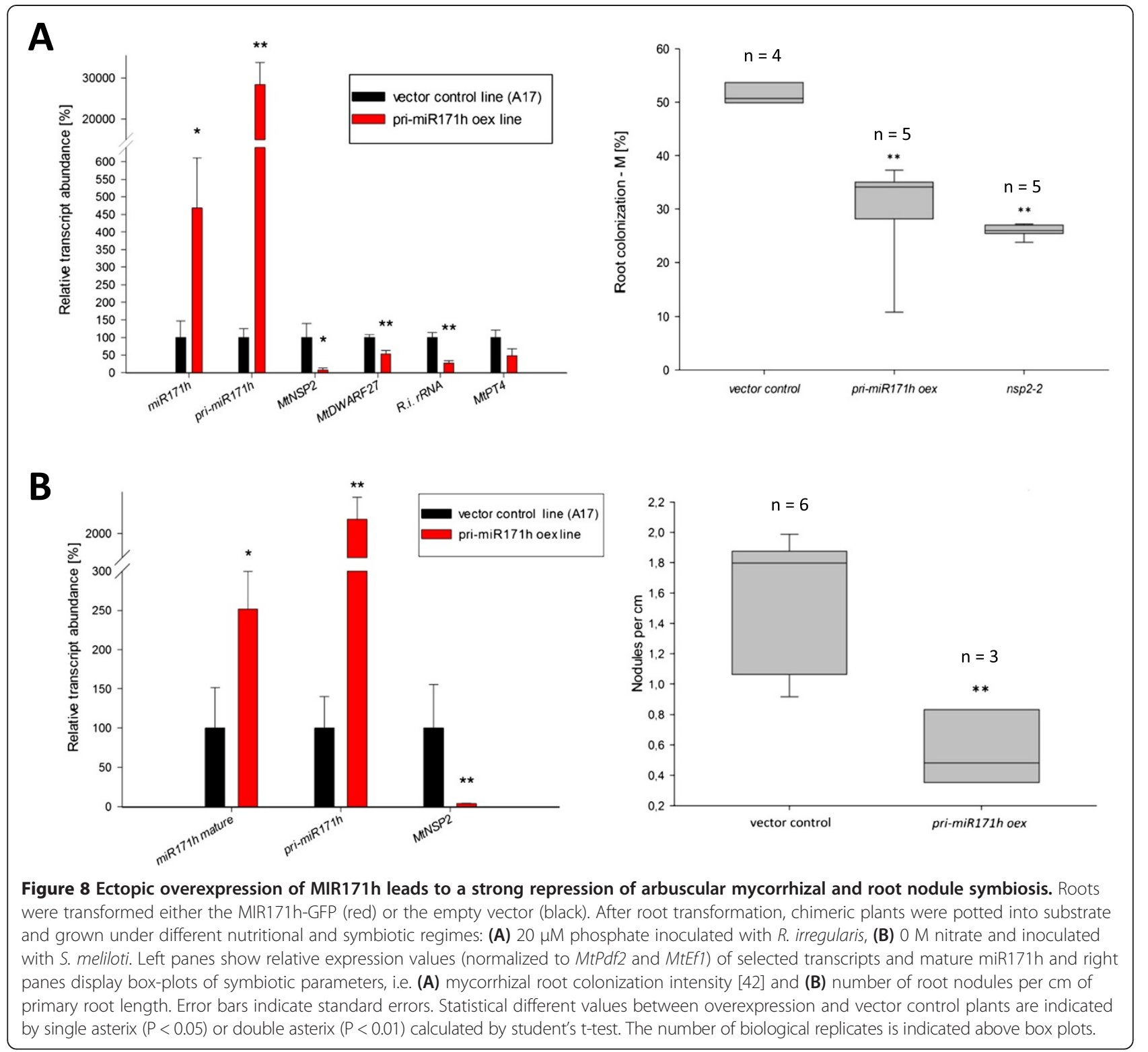

known that $\mathrm{P}_{\mathrm{i}}$ and nitrogen fertilization increases cytokinin levels of plants $[47,48]$ and elevated levels are also found in shoots and roots of mycorrhizal plants [reviewed in 35]. Based on this knowledge we hypothesized that the expression levels of miR171h at high phosphate conditions might be dependent on CRE1 as a direct effect of induced cytokinin levels in these plants. We used the cre1-1 mutant plant line to address this question. The presented results indicate that phosphate dependent regulation of miR171h and its modest induction in mycorrhizal roots is not dependent on CRE1; the latter assumption is in agreement with the fact that the cre1-1 mutation has no statistically significant effect on the mycorrhizal marker gene expression and thus mycorrhizal colonization (Figure 4C). Taken together we conclude that the miR171h expression is regulated by the phosphate status of the plant in a nitrogen dependent manner controlled by an unknown genetic factor, in addition to the previously demonstrated inducing effect of myc-LCOs [32].

Interestingly, we found elevated transcript levels of NSP2 in mycorrhizal roots (Figure 2 and Additional file 1: Figure S2) between two and six weeks post inoculation. Similar to miR171h, the expression of NSP2 is induced upon cytokinin treatment in a CRE1-dependent manner during high phosphate and low nitrogen conditions [33]. However, the involvement of CRE1 in the induction of NSP2 transcript abundance in mycorrhizal roots during low phosphate and high nitrogen conditions was not as clear as with the cytokinin treatment mentioned above 
(Figure 4B). Therefore, we assume that this up-regulation is mediated by an unknown factor and does not involve cytokinin perception per se. We cannot rule out a subtle influence CRE1 on NSP2 expression during arbuscular mycorrhizal symbiosis, though mycorrhizal colonization itself is clearly not dependent on the CRE1-pathway. The observed increase in NSP2 transcript levels is most likely the result of the observed additional promoter activity in the epidermal cell layer of mycorrhizal roots compared to non-mycorrhizal roots (Figure $5 \mathrm{E}$ and F) and which lack MIR171h promoter activities (Figure 5A). We cannot rule out the possibility that this phenomenon is due to the lack of proper loading of Argonaute proteins, the major component of the RNA induced silencing complex (RISC), with mature miR171h, but due to a lack of antibodies against M. truncatula AGO1 homologues, this assumption awaits to be tested. Additionally, NSP2 might be preferably repressed by a translational rather than posttranscriptional repression [19], which would leave the steady-state transcript abundance unchanged. However, owing to the mutually exclusive expression pattern of the miR171h-NSP2 target pair during mycorrhizal symbiosis as discussed below, we favor the aforementioned explanation. Yet, our observation of an induced NSP2 expression during mycorrhizal conditions is in contrast to the previously published results of Lauressergues et al. [32] who observed a slight decrease in NSP2 transcript levels in mycorrhizal roots. The exact cause for this discrepancy is not known, but we could show that our results are consistent over 3 independent experiments: (I) by qRT-PCR measurement of NSP2 transcript levels based on material 3 weeks post inoculation as shown in Figure 2, (II) by qRT-PCR measurement based on material from a timecourse over 6 weeks as presented in Additional file 1: Figure S2, and (III) by qRT-PCR measurement based on the parental line of the cre1-1 mutant, harboring a WT gene, 4 weeks post inoculation as shown in Figure 4. Moreover a correlation of single data points derived from individual plants based on the measurement shown in Figure 4 shows a positive correlation between MtPt4 (as a measure of arbuscule abundance) and NSP2. Additionally, we reinvestigated publically available microarray datasets, which are deposited in the Medicago Gene Expression Atlas v3 (MtGEA; http://mtgea.noble.org) using the probe Mtr.44789.1.S1_at, which corresponds to NSP2. Although the data of Gomez et al. [49] shows only a slight increase of NSP2 in mycorrhizal conditions at best, but no reduction as well, the data of Hogekamp et al. [50] shows a strong increase in the signal of the probe in response to 6 weeks of colonization by $R$. irregularis or Glomus mosseae and a strong decrease in response to $2 \mathrm{mM}$ Pi treatment compared to $20 \mu \mathrm{M}$ treated control conditions. Analysis of the supplementary data from Hogekamp et al. [50] in more detail revealed that the increase of NSP2 expression in $R$. irregularis treated roots has a $\log 2$ fold change of $0.7(\mathrm{p}$-value $=0.02)$ and in $G$. mosseae 0.84 $(\mathrm{p}$-value $=0.008)$, which represents a roughly $1.7 \times$ times induction of NSP2 expression during AM symbiosis, which is not only in agreement with our data and strongly supports our results, but also indicates that this response is conserved in symbiotic interactions with G. mosseae. As the slight induction in the data of Gomez et al. [49] is not significant and Lauressergues et al. [32] observed a reduction in NSP2 levels upon $R$. irregularis colonization it is clear that there is at least one other unknown factor involved in the regulation of the miR171h-NSP2 target pair. These might involve specific regulation by additional miR171h target genes [30,32] not investigated in this study, which might be preferably silenced by miR171h instead of NSP2 at the conditions tested by us. Future detailed analysis of their spatial expression pattern from promoter to the protein level, the latter also accounts for NSP2, might give insight into this conundrum.

When comparing the spatial distribution of the MIR171hand NSP2-promoter, it becomes evident that they predominantly show a mutually exclusive expression pattern, especially at full nutrition (cortex and root epidermis) and mycorrhizal conditions (root epidermis). Such a pattern has also been described for the miR395-SULTR2;1 miRNA target pair [51] and most likely functions to prevent expression of transcripts resulting from spontaneous promoter activity in specific tissues and cell types, a mechanism called promoter dampening [18]. That this model applies to the miR171h-NSP2 target pair is in agreement with the results from the ectopic mis-expression of miR171h by $35 \mathrm{~S}$ driven expression which leads to a drastic reduction in NSP2 transcript levels and mycorrhizal colonization (this study and [32]) as well as with the $35 \mathrm{~S}$ driven ectopic mis-expression of a miR171h-resistant NSP2 version leading to over-colonization of the root system and root apices, whereas ectopic mis-expression of the wild type NSP2 transcript, which can be cleaved by miR171h, does not [32]. This mutually exclusive expression pattern is especially pronounced at full nutrition conditions where the miR171h is strongly expressed in all root tissues, whereas the NSP2 promoter is completely inactive with the exception of the central cylinder. This assumes that the plant needs to comprehensively ensure a tight NSP2 expression control in roots of nutrient replete plants to avoid the formation of root nodule and AM symbiosis, especially at full nutrition supply. Even though we cannot exclude the possibility that miR171h induction by high phosphate fertilization has an impact on mycorrhizal repression by restricting NSP2 expression, we rule out that this effect is solely dependent on miR171h, because $n s p 2-2$ plants still respond to high phosphate treatment with repression of mycorrhizal colonization (Additional file 1: Figure S4) even though the NSP2 dependent regulation is genetically 
knocked out, indicating that other regulatory circuits act epistatically to the miR171h-NSP2 regulatory circuit. Additionally, a recent important discovery is that NSP2 interacts with another GRAS-type transcription factor RAM1, which is essential for mycorrhizal colonization and hyphopodia formation by controlling the expression of RAM2 $[15,16]$. RAM2, a glycerol-3-phosphate acyl transferase, catalyzes an important step in cutin monomer production, which seems to be an important molecule for promoting hyphopodia attachment to the plant root, and thus can also be hijacked by filamentous pathogenic fungi, e.g. Phytophthora palmivora [16]. Accordingly, it might be assumed that the mutually exclusive expression pattern of miR171h has been evolved to avoid hijacking of the NSP2 signaling pathway by pathogens, creating only a narrow conditional window to allow the formation of beneficial symbiotic interactions, presumably relying on a previous signal from a beneficial symbiotic partner. We therefore conclude that miR171h acts as a promoter dampener, which protects specific cell-types of the root at distinct nutritional and symbiotic conditions from NSP2 misexpression in a mutually exclusive expression pattern, whereas the overall transcript abundance of NSP2 in wildtype Medicago truncatula roots colonized by arbuscular mycorrhizal fungi is mainly determined by its promoter activity rather than direct cleavage by miR171h.

Other miRNA regulatory circuits often seen by miRNA-target pairs beside promoter dampening are spatial restriction and temporal regulation [18]. An exemplary spatial restriction type important in regulating root nodule development has been documented for miR169 in M. truncatula [25]. There, miR169 restricts the expression of its target MtHAP2-1 to the meristematic zone of the root nodule, to allow correct maturation of the root nodule. The data of our study suggests that spatial restriction seems to be a predominant regulatory mechanism of miR171h during root nodule development, in contrast to the segregated expression pattern discussed above. In root nodules the MIR171h and NSP2 promoter show an overlapping spatial distribution (Figure 6), with the NSP2 promoter being active in the whole nodule. MIR171h transcription and accumulation is restricted to the meristematic zone, which was confirmed by in-situ hybridization (Figure 7). Ectopic over-expression of the long miR171h primary transcript led to a significant reduction in the number of root nodules (Figure 8) and therefore could demonstrate that miR171h over-expression represses root nodule symbiosis, additional to its described capability to restrict mycorrhizal root colonization [32]. Furthermore, a recent study from De Luis et al. [36] in L. japonicus identified a homologue of miR171h, lja-miR171c, in determinate root nodules, which similarly targets LjNSP2. Its expression in nodules is dependent on the presence of Mesorhizobium loti, which indicates that the regulation of miR171h expression has a higher level of complexity than previously thought. Previous attempts to overexpress miR171h and miR171c in M. truncatula and L. japonicus failed, which could be explained by the use of the $35 \mathrm{~S}$ promoter, which seems to be diminished in arbuscule containing cells [52] and root nodules [53,54] and thus might lead to a variation of the results. Our demonstrated phenotype might be explained by a dosage dependent effect of the miR171h overexpression, because the primary transcript used in this study encodes two miR171h duplexes [31] and thus the maximum abundance has likely been doubled, balancing the lower $35 \mathrm{~S}$ activity in root nodules. Taken together, our data provides profound evidence that miR171h can directly influence the root nodule symbiosis and we hypothesize that miR171h protects the root of the plant from NSP2 mis-expression in the meristematic zone of indeterminate nodules and therefore prevents bacterial invasion of this tissue, analogous to the protection of the root tip from colonization with mycorrhizal fungi [32].

\section{Conclusion}

Our data revealed that the spatio-temporal expression of miR171h and NSP2 is tightly linked to the nutritional status of the plant and reflecting the different physiological conditions at which both types of endosymbiosis are favored by a host legume plant. Together with the results from the overexpression analysis, our data points to an important function of miR171h to integrate the nutrient homeostasis in order to safeguard the expression of NSP2 during both, arbuscular mycorrhizal and root nodule symbiosis.

\section{Methods}

\section{Plant materials and growth conditions}

Seeds of Medicago truncatula cv. Jemalong (A17), nsp2-2 and cre1-1 mutant plants were germinated as described in [28]. Seedlings were grown in a quartz sand $(0.6-1.2 \mathrm{~mm})$, vermiculite and expanded clay substrate $(7: 1: 1[\mathrm{v} / \mathrm{v}])$ with $16 \mathrm{~h}$ light $\left(25^{\circ} \mathrm{C}\right)$ and were twice a week fertilized, with half a strengths Hoagland solution containing $20 \mu \mathrm{M}$ Pi. For high Pi conditions the solution contained $1 \mathrm{mM}$ Pi and for experiments under $\mathrm{N}$ limitation $\mathrm{Ca}\left(\mathrm{NO}_{3}\right)_{2}$ and $\mathrm{KNO}_{3}$ were substituted by $\mathrm{CaCl}_{2}$ and $\mathrm{KCl}$, respectively. For mycorrhizal colonization the seedlings were inoculated with $R$. irregularis by mixing the substrate with $10 \%(\mathrm{v} / \mathrm{v})$ substrate from Allium porrum plants, which were used as host plants to propagate $R$. irregularis for at least 2 months. Plants used for analysis were harvested at time points indicated in the results section and representative parts of the roots were stained with WGA-Alexa Fluor 488 (Invitrogen) to estimate mycorrhizal colonization. Remaining roots were immediately frozen in liquid nitrogen and immediately used for total RNA extraction or stored at $-80^{\circ} \mathrm{C}$. 


\section{Molecular cloning}

The $811 \mathrm{bp}$ fragment of pri-miR171h located at the genomic position 31,405,065..31,404,255 of chromosome 3 (Mt3.5) [31] was cloned into the Cauliflower mosaic virus 35S (short 35S) driven binary vector pK7WG2D [37]. The resulting construct was named MIR171h-GFP.

The miR171h binding site of MtNSP2 and a miR171h non-cleavable sequence (scramble) were obtained by gene synthesis by Eurofins ${ }^{\oplus}\left(\mathrm{MWG}^{\oplus}\right.$ Operon) and cloned into the 35S driven binary vector pGWB455 [55] used for N-terminal mRFP protein fusions, resulting in the constructs MBS-NSP2 or MBS-mut, respectively. Both sequences contained stop codons in frame with mRFP.

Promoter regions of mtr-miR171h and MtNSP2, $900 \mathrm{bp}$ as well as 1248 bp upstream of the start codon, respectively, were amplified from wild type (Medicago truncatula cv. Jemalong) genomic DNA and cloned into the binary vector pKGWFS7 containing GFP:GUS fusion protein driven by the inserted vector.

\section{Root transformation and leaf infiltration assays}

Prior to root transformation seeds were surface sterilized (12 min conc. $\mathrm{H}_{2} \mathrm{SO}_{4}, 5$ min $6 \% \mathrm{NaClO}$ ) and germinated ( 2 days dark, $4^{\circ} \mathrm{C}, 2$ days dark, $25^{\circ} \mathrm{C}$ ) on $0.8 \%$ [w/v] water agar.

Roots of Medicago seedlings were transformed with Agrobacterium rhizogenes (ARqua1) as published by [56]. In short, roots were cut with a sterile razor blade and wounded site was dipped on a two day old $A$. rhizogenes culture, transferred to $0.8 \%[\mathrm{w} / \mathrm{v}]$ water agar in the dark for two days before transferring to selective Fahraeus medium containing $25 \mu \mathrm{g} / \mathrm{ml}$ of kanamycin and cultivated for 3 weeks in the phytotron (12 h/12 h light/dark cycle).

Tobacco leaf infiltration essay was conducted using 4 weeks old Nicotiana benthamiana plants prior to the flowering phase. All experiments were performed twice in three independent plants. Tobacco plants were saturated with water $2-4 \mathrm{~h}$ before infiltration. Agrobacterium tumefaciens (GV2260) containing the appropriate constructs was inoculated in $20 \mathrm{ml}$ YEB medium containing the appropriate antibiotics and grown for two days at $28^{\circ} \mathrm{C}$ and $250 \mathrm{rpm}$ in the dark. Cultures were pelleted in $50 \mathrm{ml}$ falcon tubes by centrifugation for $15 \mathrm{~min}$ at $4000 \mathrm{rpm}$ (Eppendorf, 5804), re-suspended in ice cold AS medium containing $10 \mathrm{mM} \mathrm{MES,} 10 \mathrm{mM} \mathrm{MgCl}_{2}$, $150 \mu \mathrm{M}$ Acetosyringon pH 5.6 and diluted to an $\mathrm{OD}_{600}$ of 0.8 to 1 with AS medium and further incubated for $3 \mathrm{~h}$ at RT under soft shaking ( $50 \mathrm{rpm})$. Infiltration was carried out as co-infiltration essay were Agrobacteria cultures containing different constructs were mixed 1:1 prior to infiltration. For single construct infiltration event, Agrobacteria, containing the respective construct, were mixed 1:1 with empty Agrobacteria. Each leaf was infiltrated with approximately $500 \mu \mathrm{l}$ into the abaxial side of the tobacco leaf according to [57]. Plants were grown under moderate light conditions for another 3 days before checked for fluorescence of the reporter proteins.

\section{Inoculation with Sinorhizobium meliloti}

Inoculation of $M$. truncatula roots with S. meliloti strain 1021 was carried out as described in [30].

\section{RNA extraction}

The RNA was extracted using the miRVANA miRNA isolation kit (Ambion) with a previous Plant Isolation Aid step (Ambion) according to the manufacturer's protocol.

\section{RT- and qRT-PCR analysis}

Quantitative RT-PCR analysis was carried out as described previously [30]. In brief, qRT-PCR analysis was performed in a $10 \mu \mathrm{l}$ reaction volume. cDNA was diluted 1:10 and $1 \mu \mathrm{l}$ was used for the reaction. The mixture contained $4 \mu \mathrm{l}$ of $0.5 \mu \mathrm{M}$ primer-pair mix and $5 \mu \mathrm{l}$ Maxima $^{\mathrm{Tm}}$ SYBR Green/ROX qPCR Master Mix (Fermentas). The qRTPCR machine, ABI Prism 7900 HT (Applied Biosystems), was used with the following reaction set up: first step $95^{\circ} \mathrm{C}$ for $10 \mathrm{~min}, 40$ cycles $95^{\circ} \mathrm{C}$ for $15 \mathrm{sec}$ and $60^{\circ} \mathrm{C}$ for $60 \mathrm{sec}$, alternately. Finally, a dissociation stage $\left(95^{\circ} \mathrm{C}\right.$ for $15 \mathrm{sec}$, $60^{\circ} \mathrm{C}$ for $15 \mathrm{sec}$ heating with ramp rate of $2 \%$ up to $95^{\circ} \mathrm{C}$ for $15 \mathrm{sec}$ ) for melting curve analysis was included. Data was collected and analyzed using SDS 2.4 (Applied Biosystems) and SigmaPlot v.12 (Systat). The threshold for Ct-values was set to 0.2 with an automatic baseline correction for all experiments. The averages of the Ct-values of $M t E F 1-\alpha, M t P D F 2$ and $M t G A P D H$ were used to calculate the housekeeping gene-index (HK-index) for normalization of transcript abundance. Functional symbiotic structures where determined by measuring the MtPt4 transcript abundance [3] and the relative colonization level was determined by measuring the RiTEF transcript abundance [41].

\section{In situ hybridization with Digoxygenin (DIG) -labeled LNA probes}

The In situ hybridization was carried out as described previously [30]. Before embedding the roots and nodules into paraffin they were fixed under vacuum using fixative (4\% paraformaldehyde in PBS, $\mathrm{pH} 7$ ). LNA probes against mtr-miR171h were custom designed by Exiqon.

\section{GUS staining and root sectioning}

$\beta$-glucuronidase (GUS) activity was used to assay miR171h and NSP2 promoter activity as described in $[58,59]$. Plants were grown under different conditions, as described earlier and harvest after 4 wpi. Shoots were removed and roots were incubated in GUS staining buffer containing $100 \mathrm{mM} \mathrm{NaPO}_{4} 1 \mathrm{mM} \mathrm{K}_{3}\left[\mathrm{Fe}(\mathrm{CN})_{6}\right], 1 \mathrm{mM} \mathrm{K}_{4}\left[\mathrm{Fe}(\mathrm{CN})_{6}\right]$, 
$10 \mathrm{mM}$ EDTA and 5-bromo-4-chloro-3-indolyl glucuronide, $\mathrm{pH} 7$ at $37^{\circ} \mathrm{C}$ for different time scales, as indicated. Roots were embedded in $4 \%[\mathrm{w} / \mathrm{v}]$ agarose and $40 \mu \mathrm{m}$ thick cross and longitudinal sections were obtained using the Leica VT 1000S vibratome (Bensheim, Germany).

\section{Microscopic analysis}

Images of sections were generated using an Olympus BX41 microscope with the cellP software (Olympus, Hamburg, Germany) using different zoom objectives as indicated.

Images of tobacco leafs were performed using the Stereo-Fluorescence Microscope MZ 16FA (Leica) with a PLANATO $1 \times$ objective (DC-DFC 300FX $1.3 \mathrm{MP}$, M.115 $\times$ ). Fluorescence proteins were excited with a mercury metal halide bulb laser, filtered with GFP3 filter system (exciting: 470/40 nm, barrier: 525/50 nm) for GFP fluorescence and a DsRED filter system (exciting: 545/ $30 \mathrm{~nm}$, barrier: 620/60 nm) for mRFP fluorescence. Bright-field images were taken with identical settings except no laser-beam or filters were used. Exposure time for fluorescence pictures were 9 seconds with gain 5 and zoom factor $11.4 \times$. Filters were successively shifted without changing settings. Image data were processed with the Leica LAS-AF Version 2.8.1.

\section{Determination of mycorrhizal parameters}

Mycorrhizal colonization parameters of Medicago roots were determined by stained with Alexa Fluor 488 Dye according to [60]. Roots were cleared with $10 \%[\mathrm{w} / \mathrm{v}$ ] $\mathrm{KOH}$ for $5 \mathrm{~min}$ at $90^{\circ} \mathrm{C}$. Roots were incubated at room temperature for at least $24 \mathrm{~h}$ in Alexa Fluor staining solution, containing $137 \mathrm{mM} \mathrm{NaCl}, 2.7 \mathrm{mM} \mathrm{KCl}, 12 \mathrm{mM}$ $\mathrm{Na}_{2} \mathrm{HPO}_{4} / \mathrm{KH}_{2} \mathrm{PO}_{4}$ and $0.01 \%$ [w/v] Alexa Fluor ${ }^{\circ} 488$ conjugate of wheat germ agglutinin (Invitrogen), and then washed five times prior mounting on microscopy slides. Mycorrhizal parameters defined by [42] are F\%: Mycorrhizal frequency, M\%: Mycorrhizal intensity per root, $\mathrm{m} \%$ : Mycorrhizal intensity per root fragment $\mathrm{A} \%$ : Arbuscule intensity per root, a\% Arbuscule intensity per root fragment.

\section{Protein extraction and western blotting}

Protein extraction and western blotting was carried out as described previously [61]. Protein concentration was determined using Bradford kit (Biorad) with provided $\gamma$-globulin standard following the manufacture's protocol.

Proteins were diluted to a final concentration of $1.5 \mu \mathrm{g} / \mu \mathrm{l}$ with homogenization buffer $(100 \mathrm{mM}$ HEPES, $10 \%$ [v/v] Glycerol, $5 \mathrm{mM}$ dithiothritol, 1 tablet cOmplete ${ }^{\mathrm{mm}}$ ULTRA Tablets (Roche) per $10 \mathrm{ml}$ buffer $\mathrm{pH}$ 7.5). $10 \mu \mathrm{l}$ of protein solution was mixed with $10 \mu \mathrm{l}$ of $2 \times$ SDS sample buffer (125 mM Tris, 20\% [v/v] glycerol, 4\% [w/v] SDS, 0.01\% [w/v] bromophenol blue, $40 \mu \mathrm{M}$ DTE) and incubated for
$5 \mathrm{~min}$ at $95^{\circ} \mathrm{C}$. Samples were centrifuged at $14000 \mathrm{~g}$ for $1 \mathrm{~min}\left(4^{\circ} \mathrm{C}\right)$ and then resolved on a $1 \mathrm{~mm} 12 \%$ SDS polyacrylamide gel. The semi-dry blot was assembled as described in the Immobilon-P Transfer Membrane (Millipore $^{\mathrm{Tx}}$ ) user guide. The blot was disassembled and the membrane was further used for immunostaining. Detection of GFP and mRFP was carried out using 1:2500 and 1:1000 dilutions for anti-GFP (rabbit, GenScript) and anti-mRFP (rabbit, Abcam ${ }^{\circ}$ ), respectively. As a loading control, a 1:10,000 diluted anti RubisCO antibody (rabbit, Agrisera $^{\mathrm{ru}}$ ) was added to each of the antibody solutions. The secondary anti-rabbit-alkaline-phosphatase $\left(\mathrm{Abcam}^{\circ}\right)$ antibody was diluted 1:5000. For visualization a readyto-use NBT/BCIP solution (Roche ${ }^{\circ}$ ) was used. The membrane was dried and scanned with the Epson scanner Perfection 4870 (Epson Scan 3.0 Software). RubisCO, $\mathrm{mRFP}$ and GFP were identified by comparison to a prestained protein size marker (Thermo Scientific).

\section{Additional file}

\begin{abstract}
Additional file 1: Includes the following supporting figures: Figure S1. In vivo confirmation of NSP2 gene silencing by miR171h using miR171h overexpression and mRFP sensor constructs. Co-infiltra2on of miR171h overexpression and mRFP sensor constructs. Figure S2. Expression profile of mycorrhizal marker genes, pri-miR171hand NSP2 during arbuscular mycorrhizal colonization of M. truncatula roots. Figure S3. Mycorrhizal colonization can be repressed by high phosphate in nsp2 mutants. Figure S4. The cre1-1 plant line shows a similar correlation between the relative NSP2 and MtPt4 transcript abundance like wild type plants. Figure S5. Cartoon representation summarizing the localization of the NSP2 and MIR171h promoter. Figure S6. Mycorrhizal parameters from root transformed wild type and miR171h overexpression plants compared to nsp2-2 plants.
\end{abstract}

Competing interest

The authors declare that they have no competing interests.

\section{Authors' contributions}

$\mathrm{VH}, \mathrm{EAD}, \mathrm{NG}$ and $\mathrm{AM}$ performed biological experiments. EAD and FK designed and initiated the research. All authors analyzed the data. EAD and FK wrote the manuscript. All authors read and approved the final manuscript.

\section{Acknowledgements}

The work was supported by the Max-Planck-Society. We are grateful to Florian Frugier in providing us seeds of cre $1-1$ and the respective WT controls as well as helpful comments. We would like to thank Ursula Krause for providing the anti-Rubisco antibody. Additionally, we would like to thank Daniela Zöller and Derek Nedveck for additional help in the sample preparations and Jean-Phillipe Combier, Nathan Pumplin as well as the anonymous reviewers for critical reading of the manuscript and their helpful comments.

Received: 18 February 2014 Accepted: 15 July 2014 Published: 23 July 2014

\section{References}

1. Harrison MJ: Molecular and cellular aspects of the arbuscular mycorrhizal symbiosis. Annu Rev Plant Physiol Plant Mol Biol 1999, 50:361-389.

2. Bucher M: Functional biology of plant phosphate uptake at root and mycorrhiza interfaces. New Phytol 2007, 173:11-26.

3. Javot H, Penmetsa RV, Terzaghi N, Cook DR, Harrison MJ: A Medicago truncatula phosphate transporter indispensable for the arbuscular mycorrhizal symbiosis. Proc Natl Acad Sci U S A 2007, 104:1720-1725. 
4. Pumplin N, Harrison MJ: Live-cell imaging reveals periarbuscular membrane domains and organelle location in Medicago truncatula roots during arbuscular mycorrhizal symbiosis. Plant Physiol 2009, 151:809-819.

5. Oldroyd GE: Speak, friend, and enter: signalling systems that promote beneficial symbiotic associations in plants. Nat Rev Microbio/ 2013, 11:252-263.

6. Akiyama K, Matsuzaki K, Hayashi H: Plant sesquiterpenes induce hyphal branching in arbuscular mycorrhizal fungi. Nature 2005, 435:824-827.

7. Maillet F, Poinsot V, Andre O, Puech-Pages V, Haouy A, Gueunier M, Cromer L, Giraudet D, Formey D, Niebel A, Martinez EA, Driguez H, Becard G, Denarie J: Fungal lipochitooligosaccharide symbiotic signals in arbuscular mycorrhiza. Nature 2011, 469:58-63.

8. Lin WY, Lin SI, Chiou TJ: Molecular regulators of phosphate homeostasis in plants. J Exp Bot 2009, 60:1427-1438.

9. Lopez-Raez JA, Charnikhova T, Gomez-Roldan V, Matusova R, Kohlen W, De Vos R, Verstappen F, Puech-Pages V, Becard G, Mulder P, Bouwmeester H: Tomato strigolactones are derived from carotenoids and their biosynthesis is promoted by phosphate starvation. New Phytol 2008, 178:863-874

10. Yoneyama K, Yoneyama K, Takeuchi Y, Sekimoto H: Phosphorus deficiency in red clover promotes exudation of orobanchol, the signal for mycorrhizal symbionts and germination stimulant for root parasites. Planta 2007, 225:1031-1038.

11. Umehara M, Hanada A, Yoshida S, Akiyama K, Arite T, Takeda-Kamiya N, Magome H, Kamiya Y, Shirasu K, Yoneyama K, Kyozuka J, Yamaguchi S: Inhibition of shoot branching by new terpenoid plant hormones. Nature 2008, 455:195-200

12. Liu W, Kohlen W, Lillo A, Op den Camp R, Ivanov S, Hartog M, Limpens E, Jamil M, Smaczniak C, Kaufmann K, Yang WC, Hooiveld GJ, Charnikhova T, Bouwmeester HJ, Bisseling T, Geurts R: Strigolactone biosynthesis in Medicago truncatula and rice requires the symbiotic GRAS-type transcription factors NSP1 and NSP2. Plant Cell 2011, 23:3853-3865.

13. Kalo P, Gleason C, Edwards A, Marsh J, Mitra RM, Hirsch S, Jakab J, Sims S, Long SR, Rogers J, Kiss GB, Downie JA, Oldroyd GE: Nodulation signaling in legumes requires NSP2, a member of the GRAS family of transcriptional regulators. Science 2005, 308:1786-1789.

14. Smit P, Raedts J, Portyanko V, Debelle F, Gough C, Bisseling T, Geurts R: NSP1 of the GRAS protein family is essential for rhizobial Nod factor-induced transcription. Science 2005, 308:1789-1791.

15. Gobbato E, Marsh JF, Vernie T, Wang E, Maillet F, Kim J, Miller JB, Sun J, Bano SA, Ratet P, Mysore KS, Denarie J, Schultze M, Oldroyd GE: A GRAStype transcription factor with a specific function in mycorrhizal signaling. Curr Biol 2012, 22:2236-2241.

16. Wang E, Schornack S, Marsh JF, Gobbato E, Schwessinger B, Eastmond P, Schultze M, Kamoun S, Oldroyd GE: A common signaling process that promotes mycorrhizal and oomycete colonization of plants. Curr Biol 2012, 22:2242-2246.

17. Oldroyd GE, Harrison MJ, Paszkowski U: Reprogramming plant cells for endosymbiosis. Science 2009, 324:753-754.

18. Voinnet O: Origin, biogenesis, and activity of plant microRNAs. Cell 2009, 136:669-687.

19. Brodersen $P$, Sakvarelidze-Achard L, Bruun-Rasmussen M, Dunoyer $P$ Yamamoto $Y Y$, Sieburth $L$, Voinnet $O$ : Widespread translational inhibition by plant miRNAs and siRNAs. Science 2008, 320:1185-1190.

20. Chen X: Small RNAs in development - insights from plants. Cur Opin Genet Dev 2012, 22:361-367.

21. Sunkar R, Li YF, Jagadeeswaran G: Functions of microRNAs in plant stress responses. Trends Plant Sci 2012, 17:196-203.

22. Guleria P, Mahajan M, Bhardwaj J, Yadav SK: Plant small RNAs: biogenesis, mode of action and their roles in abiotic stresses. Dev Reprod Biol 2011, 9:183-199.

23. Naqvi AR, Sarwat M, Hasan S, Roychodhury N: Biogenesis, functions and fate of plant microRNAs. J Cell Physiol 2012, 227:3163-3168.

24. Sun G: MicroRNAs and their diverse functions in plants. Plant Mol Biol 2012, 80:17-36

25. Combier JP, Frugier F, de Billy F, Boualem A, El-Yahyaoui F, Moreau S, Vernié T, Ott T, Gamas P, Crespi M, Niebel A: MtHAP2-1 is a key transcriptional regulator of symbiotic nodule development regulated by microRNA169 in Medicago truncatula. Genes Dev 2006, 20:3084-3088.

26. Boualem A, Laporte P, Jovanovic M, Laffont C, Plet J, Combier JP, Niebel A, Crespi M, Frugier F: MicroRNA166 controls root and nodule development in Medicago truncatula. Plant J 2008, 54:876-887.
27. Bazin J, Bustos-Sanmamed P, Hartmann C, Lelandais-Briere C, Crespi M: Complexity of miRNA-dependent regulation in root symbiosis. Philos Trans R Soc Lond B Biol Sci 2012, 367:1570-1579.

28. Branscheid A, Sieh D, Pant BD, May P, Devers EA, Elkrog A, Schauser L, Scheible WR, Krajinski F: Expression pattern suggests a role of MiR399 in the regulation of the cellular response to local Pi increase during arbuscular mycorrhizal symbiosis. Mol Plant Microbe Interact 2010, 23:915-926.

29. Gu M, Xu K, Chen A, Zhu Y, Tang G, Xu G: Expression analysis suggests potential roles of microRNAs for phosphate and arbuscular mycorrhizal signaling in Solanum lycopersicum. Physiol Plant 2010, 138:226-237.

30. Devers EA, Branscheid A, May P, Krajinski F: Stars and symbiosis: microRNA- and microRNA*-mediated transcript cleavage involved in arbuscular mycorrhizal symbiosis. Plant Physiol 2011, 156:1990-2010.

31. Branscheid A, Devers EA, May P, Krajinski F: Distribution pattern of small RNA and degradome reads provides information on miRNA gene structure and regulation. Plant Signal Behav 2011, 6:1609-1611.

32. Lauressergues D, Delaux PM, Formey D, Lelandais-Briere C, Fort S, Cottaz S, Becard G, Niebel A, Roux C, Combier JP: The microRNA miR171h modulates arbuscular mycorrhizal colonization of Medicago truncatula by targeting NSP2. Plant J 2012, 72:512-522.

33. Ariel F, Brault-Hernandez M, Laffont C, Huault E, Brault M, Plet J, Moison M, Blanchet S, Ichante JL, Chabaud M, Carrere S, Crespi M, Chan RL, Frugier F: Two direct targets of cytokinin signaling regulate symbiotic nodulation in Medicago truncatula. Plant Cell 2012, 24:3838-3852.

34. Gonzalez-Rizzo S, Crespi M, Frugier F: The Medicago truncatula CRE1 cytokinin receptor regulates lateral root development and early symbiotic interaction with Sinorhizobium meliloti. Plant Cell 2006, 18:2680-2693

35. Barker SJ, Tagu D: The roles of auxins and cytokinins in mycorrhizal symbioses. J Plant Growth Regul 2000, 19:144-154.

36. De Luis A, Markmann K, Cognat V, Holt DB, Charpentier M, Parniske M Stougaard J, Voinnet O: Two microRNAs linked to nodule infection and nitrogen-fixing ability in the legume Lotus japonicus. Plant Physiol 2012, 160:2137-2154.

37. Karimi M, Inze D, Depicker A: GATEWAY vectors for Agrobacteriummediated plant transformation. Trends Plant Sci 2002, 7:193-195.

38. Nakagawa T, Kurose T, Hino T, Tanaka K, Kawamukai M, Niwa Y, Toyooka K Matsuoka K, Jinbo T, Kimura T: Development of series of gateway binary vectors, pGWBs, for realizing efficient construction of fusion genes for plant transformation. J Biosci Bioeng 2007, 104:34-41.

39. Harrison MJ, Dewbre GR, Liu J: A phosphate transporter from Medicago truncatula involved in the acquisition of phosphate released by arbuscular mycorrhizal fungi. Plant Cell 2002, 14:2413-2429.

40. Breuillin F, Schramm J, Hajirezaei M, Ahkami A, Favre P, Druege U, Hause B, Bucher M, Kretzschmar T, Bossolini E, Kuhlemeier C, Martinoia E, Franken P, Scholz U, Reinhardt D: Phosphate systemically inhibits development of arbuscular mycorrhiza in Petunia hybrida and represses genes involved in mycorrhizal functioning. Plant J 2010, 64:1002-1017.

41. Helber N, Wippel K, Sauer N, Schaarschmidt S, Hause B, Requena N: A versatile monosaccharide transporter that operates in the arbuscular mycorrhizal fungus Glomus sp is crucial for the symbiotic relationship with plants. Plant Cell 2011, 23:3812-3823

42. Trouvelot A, Kough $J$, Gianinazzi-Pearson V: Mesure du taux de mycorhization VA d'un système radiculaire. Recherche des méthodes d'estimation ayant une signification fonctionnelle. In The Mycorrhizae: Physiology and Genetic. Edited by Gianinazzi-Pearson V, Gianinazzi S. Paris: INRA Presse; 1986:217-221.

43. Heckmann AB, Lombardo F, Miwa H, Perry JA, Bunnewell S, Parniske M, Wang TL, Downie JA: Lotus japonicus nodulation requires two GRAS domain regulators, one of which is functionally conserved in a non-legume. Plant Physiol 2006, 142:1739-1750.

44. Murakami Y, Miwa H, Imaizumi-Anraku H, Kouchi H, Downie JA, Kawaguchi M, Kawasaki S: Positional cloning identifies Lotus japonicus NSP2, a putative transcription factor of the GRAS family, required for NIN and ENOD40 gene expression in nodule initiation. DNA Res 2006, 13:255-265.

45. Pant BD, Musialak-Lange M, Nuc P, May P, Buhtz A, Kehr J, Walther D, Scheible WR: Identification of nutrient-responsive Arabidopsis and rapeseed microRNAs by comprehensive real-time polymerase chain reaction profiling and small RNA sequencing. Plant Physiol 2009, 150:1541-1555. 
46. Plet J, Wasson A, Ariel F, Le Signor C, Baker D, Mathesius U, Crespi M, Frugier F: MtCRE1-dependent cytokinin signaling integrates bacterial and plant cues to coordinate symbiotic nodule organogenesis in Medicago truncatula. Plant J 2011, 65:622-633.

47. Salama AMSEA, Wareing PF: Effects of mineral-nutrition on endogenous cytokinins in plants of sunflower (Helianthus-Annuus L). J Exp Bot 1979, 30:971-981.

48. Kuiper D, Schuit J, Kuiper PJC: Effects of internal and external cytokinin concentrations on root-growth and shoot to root ratio of Plantago-Major Ssp Pleiosperma at different nutrient conditions. Plant Soil 1988, 111:231-236.

49. Gomez SK, Javot H, Deewatthanawong P, Torres-Jerez I, Tang Y, Blancaflor EB, Udvardi MK, Harrison MJ: Medicago truncatula and Glomus intraradices gene expression in cortical cells harboring arbuscules in the arbuscular mycorrhizal symbiosis. BMC Plant Biol 2009, 9:10.

50. Hogekamp C, Arndt D, Pereira PA, Becker JD, Hohnjec N, Kuster H: Laser microdissection unravels cell-type-specific transcription in arbuscular mycorrhizal roots, including CAAT-box transcription factor gene expression correlating with fungal contact and spread. Plant Physiol 2011, 157:2023-2043.

51. Kawashima CG, Yoshimoto N, Maruyama-Nakashita A, Tsuchiya YN, Saito K, Takahashi H, Dalmay T: Sulphur starvation induces the expression of microRNA-395 and one of its target genes but in different cell types. Plant J 2009, 57:313-321

52. Pumplin N, Zhang X, Noar RD, Harrison MJ: Polar localization of a symbiosis-specific phosphate transporter is mediated by a transient reorientation of secretion. Proc Natl Acad Sci U S A 2012, 109:E665-E672.

53. Horvath B, Yeun LH, Domonkos A, Halasz G, Gobbato E, Ayaydin F, Miro K, Hirsch S, Sun J, Tadege M, Ratet P, Mysore KS, Ane JM, Oldroyd GE, Kalo P: Medicago truncatula IPD3 is a member of the common symbiotic signaling pathway required for rhizobial and mycorrhizal symbioses. Mol Plant Microbe Interact 2011, 24:1345-1358.

54. Auriac MC, Timmers AC: Nodulation studies in the model legume Medicago truncatula: advantages of using the constitutive EF1alpha promoter and limitations in detecting fluorescent reporter proteins in nodule tissues. Mol Plant Microbe Interact 2007, 20:1040-1047.

55. Nakagawa T, Suzuki T, Murata S, Nakamura S, Hino T, Maeo K, Tabata R, Kawai T, Tanaka K, Niwa Y, Watanabe Y, Nakamura K, Kimura T, Ishiguro S: Improved gateway binary vectors: High-performance vectors for creation of fusion constructs in Transgenic analysis of plants. Biosci Biotech Bioch 2007, 71:2095-2100.

56. Boisson-Dernier A, Chabaud M, Garcia F, Becard G, Rosenberg C, Barker DG Agrobacterium rhizogenes-transformed roots of Medicago truncatula for the study of nitrogen-fixing and endomycorrhizal symbiotic associations. Mol Plant Microbe Interact 2001, 14:695-700.

57. Vaucheret $\mathrm{H}$ : Promoter-dependent trans-inactivation in transgenic tobacco plants - kinetic aspects of gene silencing and gene reactivation. Cr Acad Sci lii-Vie 1994, 317:310-323.

58. Jefferson RA, Bevan M, Kavanagh T: The use of the Escherichia-Coli beta-glucuronidase gene as a gene fusion marker for studies of gene-expression in higher-plants. Biochem Soc T 1987, 15:17-18.

59. Jefferson RA, Kavanagh TA, Bevan MW: GUS fusions: beta-glucuronidase as a sensitive and versatile gene fusion marker in higher plants. Embo $J$ 1987, 6:3901-3907.

60. Grace C, Stribley DP: A safer procedure for routine staining of vesiculararbuscular mycorrhizal fungi. Mycol Res 1991, 95:1160-1162.

61. Devers EA, Teply J, Reinert A, Gaude N, Krajinski F: An endogenous artificial microRNA system for unraveling the function of root endosymbioses related genes in Medicago truncatula. BMC Plant Biol 2013, 13:82.

doi:10.1186/s12870-014-0199-

Cite this article as: Hofferek et al:: MiR171h restricts root symbioses and shows like its target NSP2 a complex transcriptional regulation in Medicago truncatula. BMC Plant Biology 2014 14:199.

\section{Submit your next manuscript to BioMed Central and take full advantage of:}

- Convenient online submission

- Thorough peer review

- No space constraints or color figure charges

- Immediate publication on acceptance

- Inclusion in PubMed, CAS, Scopus and Google Scholar

- Research which is freely available for redistribution

Submit your manuscript at www.biomedcentral.com/submit
C Biomed Central 